\title{
Trastuzumab inhibits the growth of human gastric cancer cell lines with HER2 amplification synergistically with cisplatin
}

\author{
SUN YOUNG KIM ${ }^{1 *}$, HWANG PHILL KIM ${ }^{2 *}$, YU JUNG KIM ${ }^{1}$, DO YOUN OH ${ }^{1}$, SEOCK-AH IM ${ }^{1,2}$, \\ DONGSOON LEE ${ }^{2,3}$, HYUN-SOON JONG ${ }^{2}$, TAE-YOU KIM ${ }^{1,2}$ and YUNG-JUE BANG ${ }^{1,2}$ \\ ${ }^{1}$ Department of Internal Medicine, ${ }^{2}$ Cancer Research Institute, ${ }^{3}$ Department of Laboratory Medicine, \\ Seoul National University College of Medicine, 28 Yongon-dong, Chongro-gu, Seoul 110-799, Korea
}

Received July 3, 2007; Accepted August 27, 2007

\begin{abstract}
HER2 has been found to be amplified in 10-20\% of gastric cancers, and is correlated with poor outcome. The aims of this study were to recognize HER2 amplification in gastric cancer cell lines via fluorescence in situ hybridization and to evaluate the growth inhibitory effect of trastuzumab in HER2-amplified cell lines. To elucidate the mechanism of the growth inhibition, we performed cell cycle analysis and immunoblotting of downstream molecules. We also conducted drug interaction studies of trastuzumab with other chemotherapeutic agents. HER 2 amplification was newly identified only in SNU-216 cells, and trastuzumab moderately inhibited the growth of SNU-216 cells and positive controls. Trastuzumabmediated G1 arrest occurred with increased expression of p27 ${ }^{\mathrm{KIP} 1}$ and decreased cyclins. Phosphorylation of HER2 and downstream molecules, STAT3, AKT, and ERK, was also inhibited by trastuzumab. Treatment of SNU-216 cells with trastuzumab plus cisplatin resulted in a synergistic inhibitory effect, whereas treatment of SNU-216 cells with trastuzumab plus 5-FU, or trastuzumab plus oxaliplatin produced an additive effect. These results suggest that trastuzumab combined with chemotherapeutic agents can be active against gastric cancer with HER2 amplification.
\end{abstract}

\section{Introduction}

Gastric cancer is the most common neoplasm and is the second most common cause of cancer-related death in the Republic of Korea. Although the survival of gastric cancer patients has improved in recent years due to early detection, a large proportion of patients still present with metastatic or recurrent disease. Although gastric cancer is relatively chemo-

Correspondence to: Professor Yung-Jue Bang, Department of Internal Medicine, Seoul National University College of Medicine, 28 Yongon-dong, Chongro-gu, Seoul 110-799, Korea

E-mail: bangyj@snu.ac.kr

${ }^{*}$ Contributed equally

Key words: stomach neoplasms, HER2, drug synergism, p27, cisplatin sensitive, a median survival beyond 1 year has rarely been achieved in any clinical trials with combination cytotoxic chemotherapy. Therefore, more effective and tolerable treatment strategies will be needed to improve clinical outcomes.

The HER2/neu proto-oncogene product is a potent oncoprotein in gastric cancer. Trastuzumab, a recombinant humanized anti-HER2 monoclonal antibody, has been shown to be active as a single agent and to be synergistic with other cytotoxic agents in breast cancer cell lines (1). Subsequent clinical trials revealed a clinical benefit of trastuzumab in HER2-overexpressed or amplified breast cancer patients (2). In the case of gastric cancer, HER2 overexpression and amplification have also been documented in 10-20\% of patients, and are known to be associated with poor outcome and distant metastasis (3-5). The clinical efficacy of trastuzumab in HER2amplified gastric cancer, however, has not been established, and synergism between trastuzumab and chemotherapeutic agents has not been demonstrated in gastric cancer cell lines.

The present study aims to identify the presence and the degree of HER2 amplification in several gastric cancer cell lines, and to evaluate the growth inhibitory effect of trastuzumab alone or in combination with other chemotherapeutic agents in the HER2-amplified cell lines. We also examined the expression of downstream molecules in HER2initiated signal transduction pathways and the alteration of the cell cycle-regulatory molecules in trastuzumab-treated cells to elicit the mechanism of growth inhibition.

\section{Materials and methods}

Reagents. Trastuzumab was kindly provided by Roche Korea Co., Ltd. (Seoul, Korea). To explore the interaction between trastuzumab and other chemotherapeutic agents, we used cisplatin, oxaliplatin, and 5-fluorouracil (5-FU). Oxaliplatin was kindly provided by Sanofi-Aventis Korea Co., Ltd. (Seoul, Korea); 5-FU was obtained from Ildong Pharmaceutical Co., Ltd. (Seoul, Korea); and cisplatin was purchased from Choongwae Co., Ltd. (Seoul, Korea).

Cell culture. Eleven human gastric adenocarcinoma cell lines (SNU gastric carcinoma cell lines) have been established in our institute (6). Ten of them (SNU 1, 5, 16, 216, 484, 601, $620,638,668$, and 719) were supplied by the Korean Cell Line Bank (Seoul, Korea). Another human gastric cancer cell 
line, N87, and a human breast cancer cell line, SK-BR-3, were purchased from American Type Culture Collection (Manassas, VA, USA). N87 and SK-BR-3 are cell lines in which HER2 gene amplification has been demonstrated $(7,8)$ and they were used as the positive controls. All cell lines were maintained in RPMI-1640 culture media (WelGENE Inc., Daegu, Korea) supplemented with $10 \%$ fetal bovine serum in a humidified atmosphere containing $5 \% \mathrm{CO}_{2}$ at $37^{\circ} \mathrm{C}$.

Fluorescence in situ hybridization (FISH). Each prepared cell suspension was dropped on glass slides and dried. The slides were incubated in protease solution (pepsin $50 \mu \mathrm{g} / \mathrm{ml}$ in $0.01 \mathrm{~N}$ $\mathrm{HCl}$ ) at $37^{\circ} \mathrm{C}$, treated with $10 \%$ buffered paraformaldehyde, and dehydrated through an ethanol series. Codenaturation and hybridization of probe and cellular DNA was performed using a hybridizer (Dako, Glostrup, Denmark) according to the protocol of the manufacturer. The SpectrumOrange-labeled HER2/neu probe and the SpectrumGreen chromosome 17 centromeric alpha-satellite probe were used. FISH was performed using PathVysion HER2 DNA Probe Kit (Vysis, Downers Grove, IL, USA).

Southern blot analysis. Cell suspensions were homogenized in an extraction buffer containing $10 \mathrm{mM}$ Tris- $\mathrm{HCl}, \mathrm{pH} 8.0$, 0.1 M EDTA, pH 8.0, 0.5\% SDS, and $20 \mu \mathrm{g} / \mathrm{ml}$ RNase, and were incubated for $1 \mathrm{~h}$ at $37^{\circ} \mathrm{C}$. Lysis buffer (6.0 M guanidine thiocyanate, 0.1 M sodium acetate, $\mathrm{pH}$ 5.0) was added and samples were incubated for $1 \mathrm{~h}$ at room temperature with gentle shaking. High molecular-weight DNA was purified by phenol/chloroform extraction and ethanol precipitation. The purified DNA $(10 \mu \mathrm{g})$ sample was digested with EcoRI and electrophoresed on a $0.8 \%$ agarose gel. Gel-fractionated DNA was then transferred onto nylon membrane and hybridized with 1.6- and 12-kb human HER2 cDNA fragments labeled with ${ }^{32} \mathrm{P}-\mathrm{dCTP}$ at $60^{\circ} \mathrm{C}$. Autoradiography was performed, and the intensities of the hybridization bands were quantified.

Cell growth inhibition assay. All cell lines were seeded at a density of $3 \times 10^{3}$ cells in 96-well culture plates and cultured in an incubator for $24 \mathrm{~h}$. The cells were then treated with $0.01,0.1,1,10$, and $100 \mu \mathrm{g} / \mathrm{ml}$ of drugs (trastuzumab, 5-FU, cisplatin, and oxaliplatin). Seventy-two hours later, $100 \mu \mathrm{g}$ tetrazolium dye [3-(4, 5-dimethylthiazolyl-2)-2, 5-diphenyltetrazolium bromide, MTT] (Sigma Chemical, St. Louis, MO, USA) was added to each well, followed by incubation for $4 \mathrm{~h}$ for reduction of the dye. After treatment with demethyl sulfoxide, absorbance of the converted dye in the living cells was measured at a wavelength of $570 \mathrm{~nm}$.

Dose-response curves of 5-FU, cisplatin, and oxaliplatin were also obtained based on the relative absorbance compared with untreated controls. The $\mathrm{IC}_{20}$, defined as the inhibitory concentration of a drug that produced $20 \%$ cell growth inhibition compared with untreated cells, was calculated from each dose-response curve to perform isobologram analysis. Despite the fact that the $\mathrm{IC}_{50}$ has been adopted in previous isobolgram analyses, we used the $\mathrm{IC}_{20}$ because the maximum concentration of trastuzumab induced cell death in $<30 \%$ of controls in SNU cell lines. To evaluate the interaction between trastuzumab and other agents, $\mathrm{IC}_{20}$ values were derived from dose-response curves of each combination of trastuzumab at doses of $0.001,0.01$, and $0.1 \mu \mathrm{g} / \mathrm{ml}$, and other agents at doses of $0.01,0.1,1,10$, and $100 \mu \mathrm{g} / \mathrm{ml}$.

Flow cytometry. We planned to select one of the gastric cancer cell lines with HER2 amplification to perform cell cycle analysis. After incubation with trastuzumab for $72 \mathrm{~h}$ at the concentration of $0.1,1$, and $10 \mu \mathrm{g} / \mathrm{ml}$, cells were centrifuged at $3000 \mathrm{rpm}$ for $5 \mathrm{~min}$, fixed in $75 \%$ alcohol and kept at $-20^{\circ} \mathrm{C}$. The samples were dissolved in $10 \mu \mathrm{l}$ of $100 \mu \mathrm{g} / \mathrm{ml} \mathrm{RNase}$ and incubated at $37^{\circ} \mathrm{C}$ for $10 \mathrm{~min}$. After treatment with propidium iodide, the relative DNA content per cell was obtained by measuring the fluorescence of DNA.

Western blot analysis. Cells with HER2 amplification were incubated with trastuzumab at $0.1,1$, and $10 \mu \mathrm{g} / \mathrm{ml}$ in serumfree media. After 24 and $48 \mathrm{~h}$, cells were treated with lysis buffer: $50 \mathrm{mM}$ Tris- $\mathrm{HCl}(\mathrm{pH} 7.5), 1 \% \mathrm{NP}-40,0.1 \% \mathrm{Na}$ deoxycholate, $150 \mathrm{mM} \mathrm{NaCl}, 50 \mathrm{mM} \mathrm{NaF}, 1 \mathrm{mM}$ sodium pyrophosphate, $1 \mathrm{mM}$ sodium vanadate, $1 \mathrm{mM}$ nitrophenol phosphate, $1 \mathrm{mM}$ benzamidine, $0.1 \mathrm{mM}$ PMSF, $0.1 \mathrm{mM}$ aprotinin, $0.1 \mathrm{mM}$ leupeptine, and $0.1 \mathrm{mM}$ pepstatin A. After the lysate was spun down, $20 \mu \mathrm{g}$ of protein was obtained from the suspension mixed for $12 \%$ sodium dodecyl sulfate polyacrylamide gel electrophoresis (SDS-PAGE) and transferred to a nitrocellulose membrane. After blocking with buffer, the membrane was incubated with primary antibodies at $4^{\circ} \mathrm{C}$ overnight. The following antibodies from Cell Signaling Technology (Beverly, MA, USA) were used to detect expression of downstream molecules of the HER2 signaling pathway: i) a rabbit monoclonal anti-phospho-HER2/ErbB2 antibody; ii) a rabbit monoclonal anti-Her2/ErbB2 antibody (p-HER2); iii) a rabbit monoclonal anti-STAT3 antibody; iv) a rabbit monoclonal anti-phospho-STAT3 antibody (pSTAT3); v) a rabbit monoclonal anti-AKT antibody; vi) a rabbit monoclonal anti-phospho-AKT antibody (p-AKT); vii) a rabbit monoclonal anti-p44/p42 ERK antibody; and viii) a rabbit monoclonal anti-phospho anti-p44/p42 ERK (p-ERK) antibody. Primary antibodies to cell cycle-regulatory molecules were also used. The primary antibodies were purchased from Santa Cruz Biotechnology (Santa Cruz, CA, USA): i) a mouse monoclonal anti-cyclin D1 antibody; ii) a mouse monoclonal anti-cyclin E antibody; iii) a mouse monoclonal anti-cyclin A antibody; and iv) a mouse monoclonal anti-p27 ${ }^{\mathrm{KIP} 1}$ antibody.

Isobolograms. The dose-response interactions between trastuzumab and other cytotoxic agents at the point of $\mathrm{IC}_{20}$ were evaluated by constructing 'an envelope of additivity' using the isobologram method initially described by Steel and Peckham $(9,10)$. As mentioned above, we used the isobologram at the point of $\mathrm{IC}_{20}$. If the plotted experimental $\mathrm{IC}_{20}$ concentration of a drug combination lies within the area surrounded by isoeffective curves, referred to as 'the envelope of additivity', the combination is regarded as additive. A combination that gives experimental data points to the left of the envelope of additivity can be described as synergistic.

\section{Results}

Amplification of HER2 in gastric cancer cell lines. HER2 amplification was examined in 11 cell lines, including SK- 


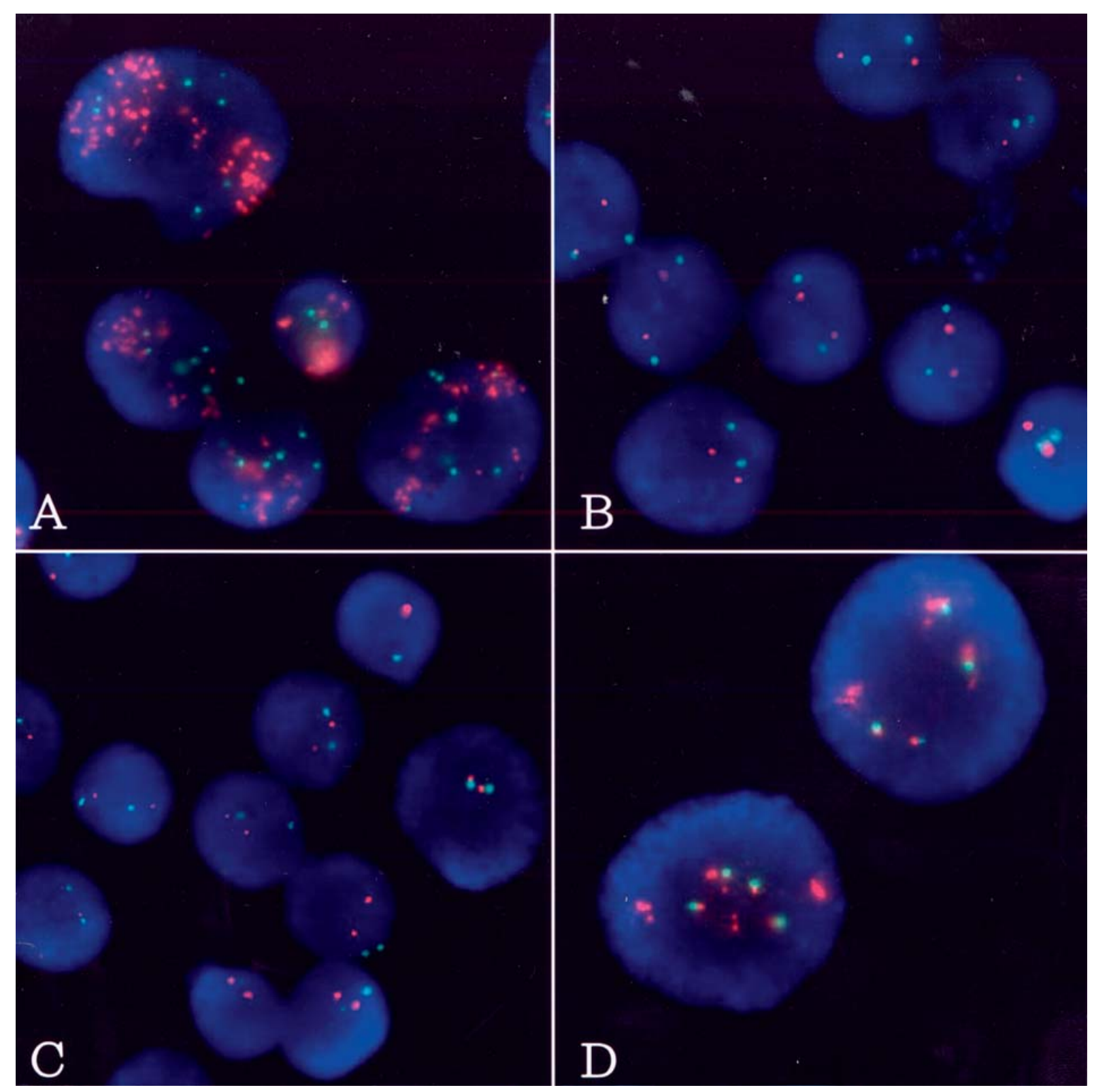

Figure 1. Fluorescence in situ hybridization (FISH) of gastric cell lines and SK-BR-3. Hybridization of prepared cell lines with HER2/neu probe (orange color) and a chromosome 17 centromere probe (green color) was performed, using a PathVision HER2 DNA probe kit, as described in Materials and methods. HER2 amplification is shown in SK-BR-3 (A) and SNU-216 (D); copy numbers of HER2 and chromosome 17 are balanced without amplification in SNU-216 (B) and SNU-219 (D).

BR-3, the positive control, using FISH analysis. Cell lines were considered to be amplified if they had a copy number ratio of HER2/neu per chromosome 17 centromere $>2.0$ (Fig. 1). As expected, SK-BR-3 showed a high level of amplification (copy number ratio, 4.9). Among SNU gastric cell lines, only SNU-216 showed HER2 amplification with a copy number ratio of 4.34, while none of the other SNU gastric cancer cell lines had a copy number ratio $>2.0$ (ranging from 0.17 to 1.32 ). The FISH results corresponded with those of Southern blot analysis, which revealed a high level of HER2 amplification only in the SNU-216 cell line among all of the SNU gastric cancer cell lines (data not shown).

Effect of trastuzumab in gastric cancer cell lines. As shown in Fig. 2A, MTT assays were performed to evaluate the cytotoxic activity of trastuzumab in gastric cancer cell lines and SK-BR-3. The result indicated that trastuzumab exerted its activity selectively in HER2-amplified gastric cancer cell lines. Cellular growth was suppressed in the SNU-216, SKBR-3, and N87 lines, all of which were HER2-amplified cell lines. At the maximum concentration of $100 \mu \mathrm{g} / \mathrm{ml}$, the cell

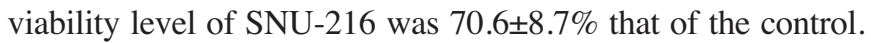
In the SK-BR-3 and N87 lines, the percentages of viable cells were $58.2 \pm 8.2 \%$ and $57.1 \pm 7.0 \%$, respectively. It was notable that the portion of viable cells in the SNU-216 line decreased to $<80 \%$ that of control cells, even in the lowest concentration of trastuzumab $(0.01 \mu \mathrm{g} / \mathrm{ml})$. Growth of other cell lines without HER2 amplification (SNU-1, SNU-5, and SNU-484), however, was maintained at viable levels $>80-90 \%$ that of the control. At $100 \mu \mathrm{g} / \mathrm{ml}$ of trastuzumab, cell viabilities were $108.9 \pm 23.9 \%$, $95.1 \pm 6.5 \%$, and $97.0 \pm 7.4 \%$ in SNU-1, SNU-5, and SNU-484 cells, respectively. 
A

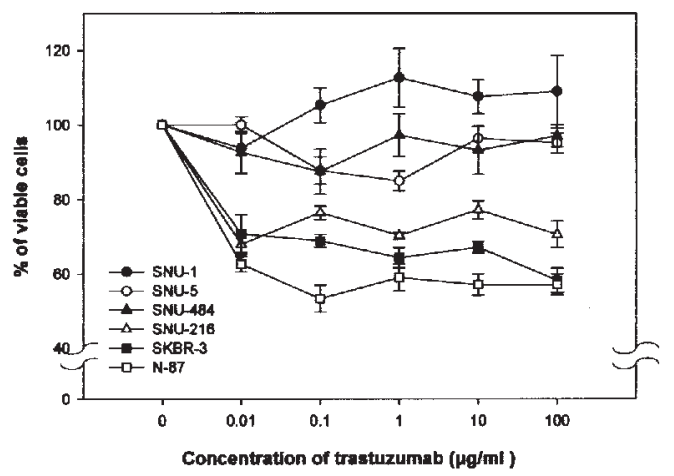

B

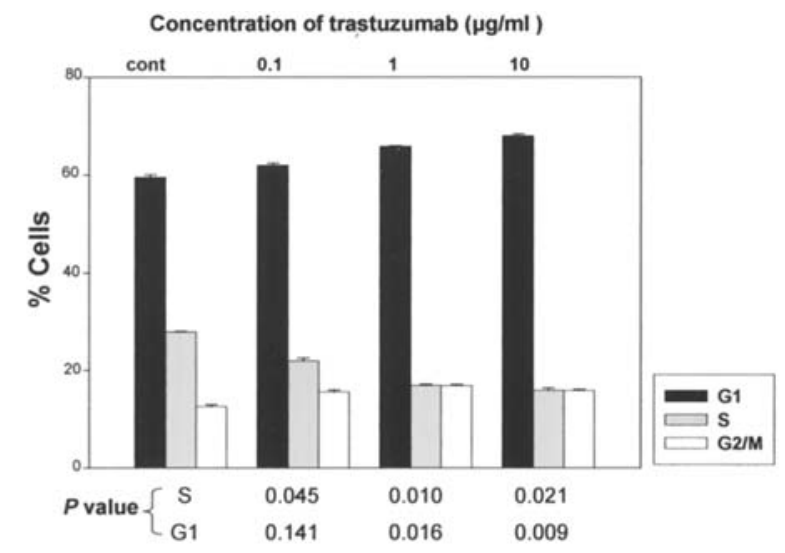

Figure 2. (A) Dose-response curves of trastuzumab in gastric cancer cell lines and positive controls (SK-BR-3, N87). Exponentially growing cells were treated with the indicated concentrations of trastuzumab for $72 \mathrm{~h}$. Cell growth inhibition was analyzed by MTT assay, as described in Materials and methods. Ratios of mean absorbance of wells containing drug over mean absorbance of drug-free wells were plotted against different concentrations of tested drugs. The assay was performed using six replicates. Points, mean; bars, \pm SE. (B) Cell cycle analysis of SNU-216 cells $72 \mathrm{~h}$ after trastuzumab treatment. After cells had been fixed and stained with propidium iodide, the DNA content was measured by flow cytometry. Cell cycle distribution was analyzed using a FACSCalibur flow cytometer (Becton Dickinson, Franklin Lakes, NJ). The population percentages in the $\mathrm{G} 1$ and $\mathrm{S}$ phase from independent duplicate cultures were plotted. The fraction of cells in the G1 phase increased with the escalation of the concentration of trastuzumab, while the fraction of cells in the S phase decreased. Columns, percentages of cells in G1, S, and G2/M for each group; bars, SD between duplicate cultures. Statistics were calculated by Student's unpaired t-test.

Effect of trastuzumab on the cell cycle of the HER2-amplified cell line. In flow cytometry analysis of SNU-216 cells, trastuzumab induced cell cycle arrest at the G1 phase in a dose-dependent manner (Fig. 2B). The cells treated with $10 \mu \mathrm{g} / \mathrm{ml}$ of trastuzumab increased the fraction of $\mathrm{G} 1$ phase cells to $68.0 \%$, compared to $59.55 \%$ in controls, and this difference was statistically significant $(\mathrm{P}=0.009)$. At the same time, trastuzumab also decreased the fraction of cells in the $\mathrm{S}$ phase in a dose-dependent manner. The initial $\mathrm{S}$ phase fraction was $27.9 \%$, but decreased to $15.8 \%$ when exposed to $10 \mu \mathrm{g} / \mathrm{ml}$ of trastuzumab $(\mathrm{P}=0.02)$. Because the DNA histogram at any concentration of trastuzumab showed no sub-G1 peak, apoptosis was not thought to be induced by trastuzumab in SNU-216 cells.

Expression pattern of downstream molecules of the HER2initiated signal transduction pathway and cell cycle-regulatory
A

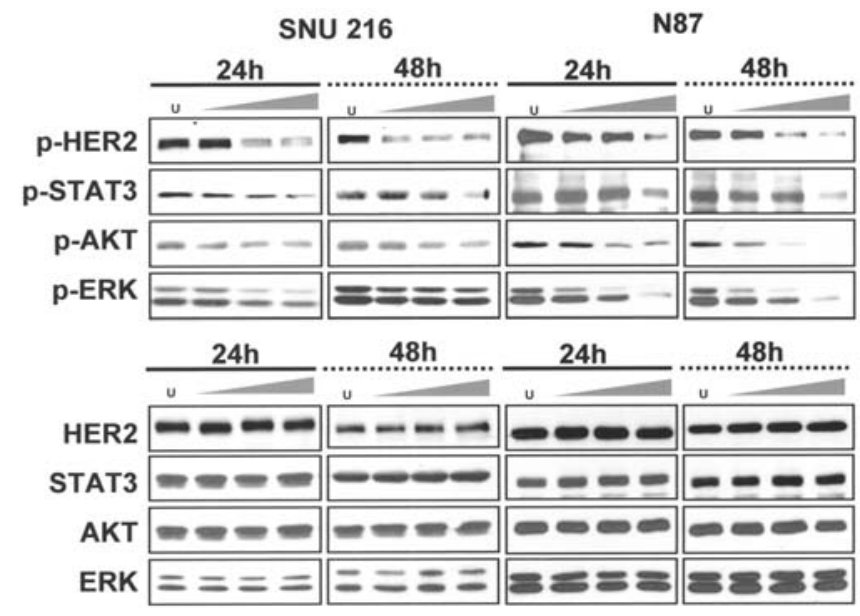

B

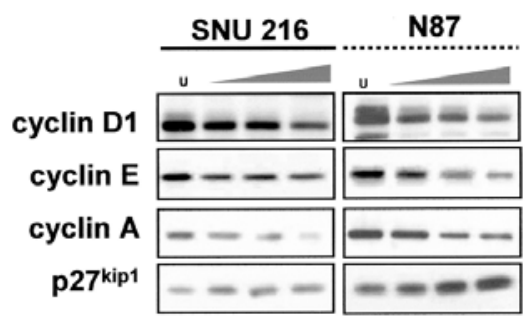

Figure 3. Western blot analysis of HER2 signaling and cell cycle-regulators in the SNU-216 and N87 cell lines. Cells were harvested after being incubated with the indicated concentrations of trastuzumab for 24 and 48 h. (A) Phosphorylated forms of HER2, AKT, ERK, and STAT3 were down-regulated without changing the expression of total proteins. (B) Expression of cyclin D1, cyclin E and cyclin A were decreased by trastuzumab, while the level of $\mathrm{p} 27^{\mathrm{KIPl}}$ was increased.

molecules. To examine the alteration of the signal transduction pathway by trastuzumab, we performed Western immunoblot assays of HER2 signaling pathways in trastuzumab-treated gastric cancer cell lines with HER2 amplification. As expected, treatment with trastuzumab produced a significant reduction of HER2 phosphorylation in the SNU-216 and N87 cell lines. Phosphorylated forms of downstream molecules of trastuzumab, p-STAT, p-AKT, and p-ERK, also decreased in a dose-dependent manner in both cell lines, but the decreasing pattern was more prominent in the N87 cell line than in the SNU-216 line (Fig. 3A). For N87, in particular, the band of p-AKT nearly disappeared $48 \mathrm{~h}$ after treatment with $10 \mu \mathrm{g} / \mathrm{ml}$ of trastuzumab.

In Western immunoblot assays of cell cycle-regulatory molecules, trastuzumab reduced expression of cell cycle inducer proteins, cyclin A, D1, and E, in a dose-dependent manner, while the protein level of $\mathrm{p} 27^{\mathrm{KIP} 1}$, a cyclin-dependent kinase inhibitor, increased with elevated concentrations of trastuzumab.

Interaction between trastuzumab and other chemotherapeutic agents. SNU-216 cells were treated with various concentrations of trastuzumab and other chemotherapeutic agents to assess drug interaction. We used active agents for gastric cancer, 5-FU, and two platinum-derivatives, cisplatin and oxaliplatin. Isobolograms and data points from the combination of trastuzumab and other drugs are presented in Fig. 4. Because the 
A

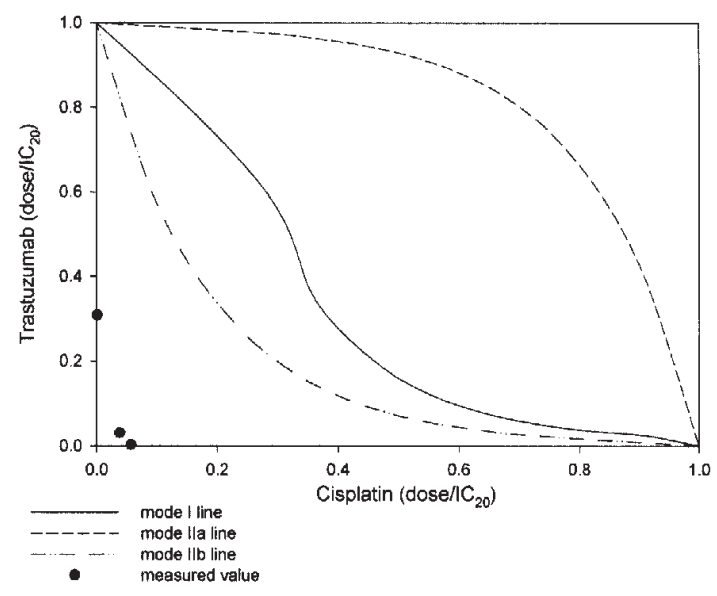

B

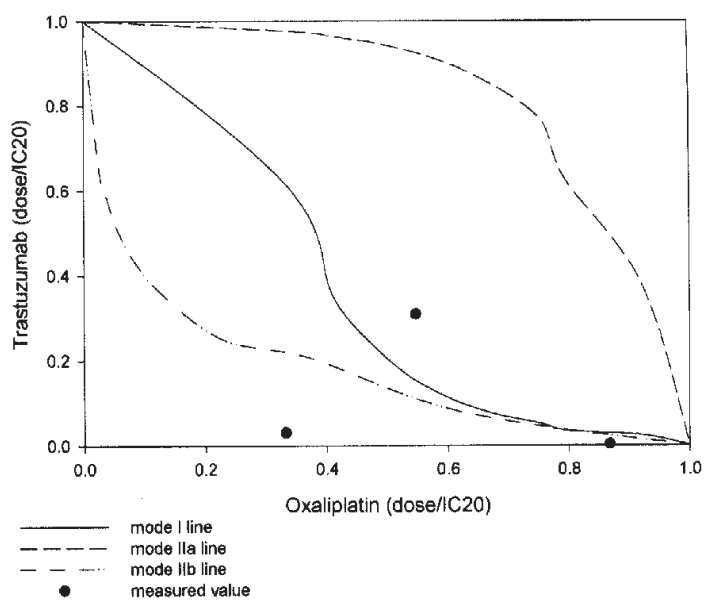

C

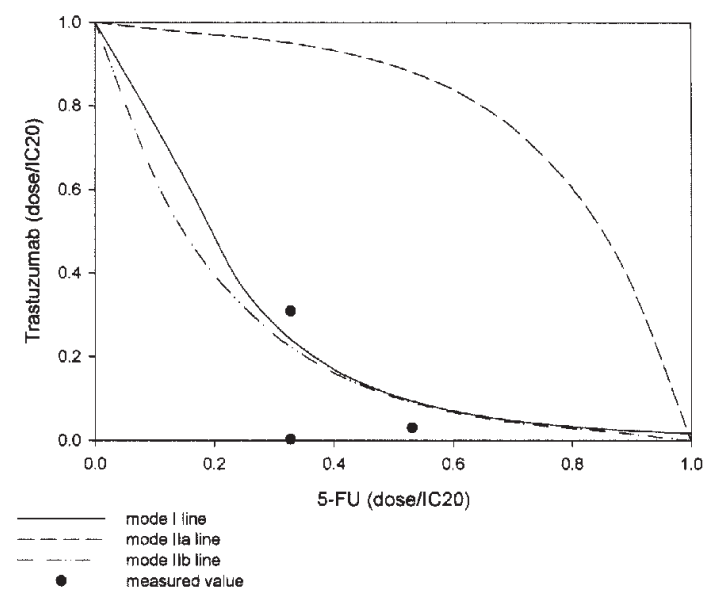

Figure 4. Isobologram of the SNU-216 line consisting of three lines and several data points. Lines representing Mode I (solid line), IIa (dotted line), and IIb (perforated line) were derived from the dose-response curves of each of the drugs. Dots represent measured values, which were derived from the dose-response curves of combinations of trastuzumab and chemotherapeutic agents. (A) All plotted measured values of the combination of trastuzumab and cisplatin were placed on the left side of the 'envelope of additivity', so cisplatin was considered to have a purely synergistic growth inhibition effect with trastuzumab. (B and C) In the cases of 5-FU and oxaliplatin, respectively, some measured values were placed inside of the envelope, which indicated that there were additive effects of 5-FU with trastuzumab, or oxaliplatin with trastuzumab.

$\mathrm{IC}_{20}$ value of trastuzumab was $0.32 \pm 0.16 \mu \mathrm{g} / \mathrm{ml}$, we chose concentrations of a lower level than the $\mathrm{IC}_{20}$ such as 0.001 , 0.01 , and $0.1 \mu \mathrm{g} / \mathrm{ml}$ for analysis of drug interaction. When cisplatin and trastuzumab were used in combination, all data points fell on the left side of the envelope, so cisplatin was considered to produce a synergistic effect with trastuzumab (Fig. 4A).

In contrast, the inhibitory effect was additive or synergistic according to the concentration when trastuzumab was combined with 5-FU or oxaliplatin, because the data points were located on both the left side and inside of the envelope (Fig. 4B and C).

\section{Discussion}

HER2 is a well-known oncoprotein that functions as a receptor tyrosine kinase that affects a number of signal programs leading to cell proliferation, protection from apoptosis, differentiation, migration, and transformation. Its overexpression is observed in several human carcinomas, including breast, ovarian, gastric, colon, and non-small cell lung cancer. Trastuzumab, the new biologic agent targeting HER2, is already known to be one of the most important therapeutic options in HER2-amplified breast cancers, but its clinical efficacy is still unclear in other kinds of cancer.

HER2-amplified gastric cancer is one of the most probable candidates that are likely to respond to trastuzumab, because some in vitro studies have demonstrated significant sensitivity of HER2-amplified gastric cancer cell lines to the drug. Tanner et al demonstrated high levels of HER2 mRNA, protein, and HER2 gene amplification of N87 and activity of trastuzumab in a xenograft model of N87 (7). Similarly, Matsui et al identified $\mathrm{N} 87$ and MKN-45P as human gastric cancer cell lines with HER 2 overexpression by using Western blot analysis, and found that trastuzumab suppressed the tumor growth of N87 cells in a murine model (11). Recently, another gastric cancer cell line, 4-1ST, was shown to have HER2 amplification in FISH analysis, and its growth was found to be suppressed by trastuzumab in a xenograft model (12).

In this study, we newly identified SNU-216 as an HER2amplified cell line among gastric cancer cell lines that were established in the Korean Cell Line Bank, at the Cancer Research Institute of Seoul National University (6). HER2 amplification was demonstrated in SNU-216 by FISH and Southern blot assay, and a cell proliferation assay with MTT showed growth inhibition by nearly $30 \%$ in the SNU-216 cell line. In the positive control cell lines, N87 and SK-BR-3, trastuzumab also showed moderate activity, inhibiting cellular growth by up to $40-45 \%$. As expected, trastuzumab exerted little effect on cells without HER2 amplification. Although cytotoxic agents usually induce near-complete cell death, dose-response curves of trastuzumab in HER2-amplified cells showed a plateau $>60-70 \%$ that of controls, even at the highest dose levels. These results are consistent with those of previous in vitro studies, in which 30-60\% growth inhibition was observed, at most, in HER2-amplified breast cancer cell lines, SK-BR-3 or BT-474. These results indicate the cytostatic rather than cytotoxic feature of trastuzumab $(13,14)$.

In our study, the anti-proliferative effect of trastuzumab on SNU-216 cells mainly came from cell cycle arrest, rather than apoptosis. Cell cycle analysis revealed that trastuzumab reduced the proportion of cells in the $S$ phase, increased those in the G1 phase, but hardly induced apoptosis. Cell 
cycle arrest of SNU-216 cells at the G1 phase is consistent with the results of the Western blot assay of cyclins and p27 ${ }^{\mathrm{KIP} 1}$. The level of expression decreased in cyclin D1, E, and A along with elevated concentrations of trastuzumab, while a cyclin-dependent kinase (Cdk), p27 ${ }^{\mathrm{KIP} 1}$, was induced in both the SNU-216 and N87 cell lines. Although the working sequences of cyclin D, E, and A differ, they have a common function of enhancing progression into the $\mathrm{S}$ phase from the G1 phase. Among them, cyclin E binds to Cdk2 and forms the Cdk2/ Cyclin E complex, a target of p27 KIP1. It is known that increased $\mathrm{p} 27^{\mathrm{KIP} 1}$ causes proliferating cells to exit the cell cycle, and expression of $\mathrm{p} 27^{\mathrm{KIP} 1}$ is inversely correlated with HER 2 overexpression. Recently, the induction of $\mathrm{p} 27^{\mathrm{KIP} 1}$ has been shown to be an essential event for growth inhibition and cell cycle arrest by trastuzumab in breast cancer cell lines (15). In SK-BR-3 and BT-474, it was demonstrated that p27KIP1 was up-regulated by anti-HER2 antibodies, and the level of expression paralleled the magnitude of tumor growth inhibition (15). We showed that $\mathrm{p} 27^{\mathrm{KIP} 1}$ was also induced by trastuzumab in SNU-216 and N87 cells, so these results are in agreement with the previous data derived from breast cancer cell lines.

The mechanism of trastuzumab in breast cancer cells is known to be its binding to HER2 protein and impeding heterodimerization with other epidermal growth factor receptors, which results in suppressed autophosphorylation of HER2, followed by the deactivation of downstream signal pathways. In SK-BR-3 and BT-474, trastuzumab was shown to inhibit the phosphorylation of AKT and ERK, which was in accordance with the growth inhibitory effect $(8,16)$. We postulated that trastuzumab works in the same manner in gastric cancer cell lines with HER2 amplification, so phosphorylation of HER2 and downstream molecules related to the pathways would be inhibited by trastuzumab. HER2 and these downstream molecules, which include STAT3, AKT, and ERK, were tested by immunoblotting, which showed that the amount of phosphorylated HER2 protein decreased with an elevated dose of trastuzumab in both gastric cancer cell lines, N87 and SNU-216, and the expression of downstream molecules was reduced. The pattern of decrement was more prominent in N87 cells than SNU-216 cells, especially for AKT, which explained the higher growth inhibitory effect of trastuzumab on N87 cells in the MTT assay.

STAT3, AKT, and ERK pathways transmit survival signals related to both the prevention of apoptosis and the progression of the cell cycle. In particular, AKT and ERK are known to be involved in the down-regulation and degradation of p27 ${ }^{\mathrm{KIP} 1}$ by HER2, so trastuzumab may exert its activity on the expression of $\mathrm{p} 27^{\mathrm{KIP} 1}$ by blocking AKT or ERK pathways $(15,17)$. However, it is unclear why apoptosis rarely occurred in SNU-216 cells despite the inhibition of AKT and ERK. Moulder et al showed that trastuzumab induced apoptosis in the SK-BR-3 cell line, while it only induced growth arrest of BT-474 (18). These discordant outcomes may be caused by differences in signals following the phosphorylation of AKT or ERK, or by different patterns of transcription or pharmacodynamics.

When coupled with other cytotoxic agents, the antitumor activity of trastuzumab is known to be enhanced. Therefore, chemotherapeutic agents such as taxanes, platinums, or vinorelbine are used in combination with trastuzumab in breast cancer patients $(2,19)$. In the case of platinum-derivatives, trastuzumab augments the cytotoxicity of cisplatin or carboplatin by reducing DNA repair activity following platinuminduced DNA damage in breast cancer cell lines $(20,21)$. It is also known that resistance to cisplatin can be reversed by anti-HER2 antibody in HER2-overexpressed cells (20). In this study, trastuzumab and cisplatin showed synergism in the SNU-216 cell line by the isobologram method, while the combination of oxaliplatin and trastuzumab showed an additive or synergistic effect. These inconsistent results are not fully understood, but because DNA-oxaliplatin adducts are known to avoid surveillance by mismatch repair genes, it may be that the amplified cytotoxic effect caused by the addition of trastuzumab is diluted (22).

In the case of 5-FU, our results showed that combination with trastuzumab had an additive effect in SNU-216 cells. Unlike platinum-derivatives, chemotherapeutic agents that do not have a direct DNA-damaging effect, such as taxanes and antimetabolites, have been shown to be additive with trastuzumab in breast cancer cell lines. Combination of 5-FU with trastuzumab was even antagonistic in SK-BR-3. These results were explained by decreased sensitivity to 5-FU due to the reduction of cells in the $S$ phase by trastuzumab, because the principal mechanism of the action of 5-FU is inhibition of thymidylate synthetase, which results in interference with DNA biosynthesis (23). However, incorporation into RNA, another mechanism of antitumor activity of 5-FU, occurs in a relatively nonspecific mode for the cell cycle, so 5-FU might have been less affected by trastuzumab in our experiment than in other conditions. Anyway, fluoropyrimidine is one of the most active chemotherapeutic agents in gastric cancer, so further evaluation of the interaction between fluoropyrimidine and trastuzumab is needed.

In summary, this study demonstrates that trastuzumab has an anti-proliferative effect in an HER2-amplified gastric cancer cell line, SNU-216. It is suggested that this effect results from G1 arrest, accompanied by up-regulation of p27 ${ }^{\mathrm{KIP} 1}$. Phosphorylation of HER2, as well as the downstream molecules, was inhibited by trastuzumab in gastric cancer cell lines, N87 and SNU-216. Cisplatin was shown to have synergism with trastuzumab in the SNU-216 cell line. These results provide evidence that trastuzumab could have a role in the treatment of patients with HER2-amplified gastric cancer when it is combined with cytotoxic agents. A multicenter clinical trial of trastuzumab in HER2-amplified gastric cancer patients is ongoing, so its clinical efficacy should soon be elucidated.

\section{Acknowledgements}

This study was supported by the second stage Brain Korea 21 Project in 2006.

\section{References}

1. Pegram MD, Lopez A, Konecny G and Slamon DJ: Trastuzumab and chemotherapeutics: drug interactions and synergies. Semin Oncol 27: 21-25, 2000

2. Slamon DJ, Leyland-Jones B, Shak S, et al: Use of chemotherapy plus a monoclonal antibody against HER2 for metastatic breast cancer that overexpresses HER2. N Engl J Med 344: 783-792, 2001 
3. Nakajima M, Sawada H, Yamada Y, et al: The prognostic significance of amplification and overexpression of c-met and c-erb B-2 in human gastric carcinomas. Cancer 85: 1894-1902, 1999.

4. Orita H, Maehara Y, Emi Y, Kakeji Y, Baba H, Korenaga D and Sugimachi K: c-erbB-2 expression is predictive for lymphatic spread of clinical gastric carcinoma. Hepatogastroenterology 44: 294-298, 1997.

5. Im SA, Lee KE, Nam EM, et al: The prognostic significance of the overexpression of HER-2/neu in Korean gastric carcinomas and the in vitro effects of anti-HER-2/neu antibody on cell growth in the gastric carcinoma cell lines. Cancer Res Treat 35: 109-116, 2003.

6. Ku JL and Park JG: Biology of SNU cell lines. Cancer Res Treat 37: 1-19, 2005.

7. Tanner M, Hollmen M, Junttila TT, et al: Amplification of HER-2 in gastric carcinoma: association with Topoisomerase IIalpha gene amplification, intestinal type, poor prognosis and sensitivity to trastuzumab. Ann Oncol 16: 273-278, 2005.

8. Yakes FM, Chinratanalab W, Ritter CA, King W, Seelig S and Arteaga CL: Herceptin-induced inhibition of phosphatidylinositol3 kinase and Akt is required for antibody-mediated effects on p27, cyclin D1, and antitumor action. Cancer Res 62: 4132-4141, 2002.

9. Steel GG and Peckham MJ: Exploitable mechanisms in combined radiotherapychemotherapy: the concept of additivity. Int J Radiat Oncol Biol Phys 5: 85-91, 1979.

10. Kim JH, Lee KW, Jung Y, et al: Cytotoxic effects of pemetrexed in gastric cancer cells. Cancer Sci 96: 365-371, 2005.

11. Matsui Y, Inomata M, Tojigamori M, Sonoda K, Shiraishi N and Kitano S: Suppression of tumor growth in human gastric cancer with HER2 overexpression by an anti-HER2 antibody in a murine model. Int J Oncol 27: 681-685, 2005.

12. Fujimoto-Ouchi K, Sekiguchi F, Yasuno H, Moriya Y, Mori K and Tanaka Y: Antitumor activity of trastuzumab in combination with chemotherapy in human gastric cancer xenograft models. Cancer Chemother Pharmacol 59: 795-805, 2007.

13. Normanno N, Campiglio M, De Luca A, et al: Cooperative inhibitory effect of ZD1839 (Iressa) in combination with trastuzumab (Herceptin) on human breast cancer cell growth. Ann Oncol 13: 65-72, 2002.
14. Hudziak RM, Lewis GD, Winget M, Fendly BM, Chepard HM and Ullich A: p185 $18 E R 2$ monoclonal antibody has antiproliferative effects in vitro and sensitizes human breast tumor cells to tumor necrosis factor. Mol Cell Biol 9: 1165-1172, 1989.

15. Le XF, Pruefer F and Bast RC Jr: HER2-targeting antibodies modulate the cyclin-dependent kinase inhibitor p27Kip1 via multiple signaling pathways. Cell Cycle 4: 87-95, 2005.

16. Wang CX, Koay DC, Edwards A, Lu Z, Mor G, Ocal IT and Digiovanna MP: In vitro and in vivo effects of combination of Trastuzumab (Herceptin) and Tamoxifen in breast cancer. Breast Cancer Res Treat 92: 251-263, 2005.

17. Zhou BP and Hung MC: Dysregulation of cellular signaling by HER2/neu in breast cancer. Semin Oncol 30: 38-48, 2003.

18. Moulder SL, Yakes FM, Muthuswamy SK, Bianco R, Simpson JF and Arteaga CL: Epidermal growth factor receptor (HER1) tyrosine kinase inhibitor ZD1839 (Iressa) inhibits HER2/neu (erbB2)-overexpressing breast cancer cells in vitro and in vivo. Cancer Res 61: 8887-8895, 2001.

19. Baselga J, Norton L, Albanell J, Kim YM and Mendelsohn J: Recombinant humanized anti-HER2 antibody (Herceptin) enhances the antitumor activity of paclitaxel and doxorubicin against HER2/neu overexpressing human breast cancer xenografts. Cancer Res 58: 2825-2831, 1998.

20. Pietras RJ, Fendly BM, Chazin VR, Pegram MD, Howell SB and Slamon DJ: Antibody to HER-2/neu receptor blocks DNA repair after cisplatin in human breast and ovarian cancer cells. Oncogene 9: 1829-1838, 1994.

21. Pegram MD, Konecny GE, O'Callaghan C, Beryt M, Pietras R and Slamon DJ: Rational combinations of trastuzumab with chemotherapeutic drugs used in the treatment of breast cancer. J Natl Cancer Inst 96: 739-749, 2004.

22. Cvitkovic E: Ongoing and unsaid on oxaliplatin: the hope. Br J Cancer 77 (suppl 4): 8-11, 1998.

23. Pegram M, Hsu S, Lewis G, et al: Inhibitory effects of combinations of HER-2/neu antibody and chemotherapeutic agents used for treatment of human breast cancers. Oncogene 18: 2241-2251, 1999. 